\title{
Comparative Study of Low Molecular Weight Heparin and Conventional Heparin in Acute Myocardial Infarction and Unstable Angina
}

\author{
Dr. A.Meena Kumari M.D \\ Professor of pharmacology, Guntur Medical College, Guntur.
}

\begin{abstract}
:
Objectives: To Compare low molecular weight heparin and conventional heparin in acute myocardial infarction and unstable angina.

Materials and Methods: 100 patients with symptoms of acute MI and unstable angina were included in the study.50 patients were treated with Conventional Heparin and 50 treated with low molecular weight heparin for 5 days. Patients were monitored by serial ECGs, echocardiograms, blood counts, bleeding time and clotting time and were followed every week for 6 weeks.

Results: Relief of chest pain was seen in 52\% of patients on conventional heparin and in $66 \%$ on low molecular heparin on the $2^{\text {nd }}$ day. Bleeding complications (haematemesis in 4\% and haemoptysis $2 \%$ ) occurred in patients on standard heparin while no such effects were seen in patients on low molecular heparin.

Conclusion: Low molecular weight heparin is equally effective in the treatment of acute myocardial infarction and unstable angina as that of conventional heparin with the added advantage of low complication rate and ease of administration.
\end{abstract}

Keywords: Low molecular weight heparin, conventional heparin, acute myocardial infarction, unstable angina.

\section{Introduction}

The incidence of myocardial infarction all over the world is very high especially in the developed countries. Unstable angina and non $\mathrm{Q}$ wave myocardial infarction together constitute a formidable medical problem. Remarkable recent advances in our understanding of the patho physiology of acute coronary syndrome has outpaced progress in developing novel effective therapies[1]. Anticoagulants might be beneficial in the management of Acute Myocardial Infarction to halt or slow progression and to prevent the development of new thrombus elsewhere in the coronary arterial tree, to reduce the incidence of venous thrombosis and pulmonary embolism, to diminish the formation of mural thrombi and resultant systemic emboli[2].

Intravenous heparin is highly effective in the management of patients with unstable angina but continuous intravenous infusion and careful monitoring of blood clotting by aPTT are needed[3]. Low molecular weight heparin in fixed doses subcutaneously is shown to have antithrombotic properties similar to standard heparin given intravenously with fewer bleeding complications[4],[5]. The idea of extending use of low molecular weight heparins in the treatment of acute MI and non Q MI has led to this study, to prove the clinical efficacy of low molecular weight heparins given subcutaneously[6]

\section{Patients and Methods}

100 patients above 21 years of age with symptoms of with chest pain, ECG change showing acute MI, unstable angina, non Q wave MI admitted to the coronary care unit of Government General Hospital, Guntur were included in the study.

Patients with bundle branch blocks, history of contraindications to anticoagulant therapy, pregnancy and with renal disorders were excluded.

50 patients were treated with Standard Heparin and 50 treated with low molecular weight heparin after obtaining written consent.

\section{Study design and protocol}

The patients were randomized into two groups.

Group-1.50 patients received Standard Heparin (or conventional heparin) 10000/- I.U. given stratum as a bolus intravenously followed by 5000/- I.U / $6^{\text {th }}$ hourly for five days. Among the 50 patients 20 had acute anterior MI, 15 had inferior MI, 8 had unstable angina and 7 showed non Q MI. 
Group-2. 50 patients received low molecular weight heparin or enoxaparin in fixed doses of 3500 units subcutaneously twice daily. Among 50 patients , 14 had anterior MI, 11 had inferior MI, 11 showed non Q MI and 14 had unstable angina

Drugs used were conventional heparin of Biological Evans Limited Company and Low molecular weight heparin (enoxaparin)

Patients were monitored by serial ECGs echocardiograms, blood counts, bleeding time and clotting time before starting and after stopping heparin or low molecular heparin. (Refer charts)

Thrombolytic therapy was given for suitable cases and all patients received aspirin, ranitidine, intravenous nitroglycerine. Beta blockers antihypertensive agents, anxiolytics and antidiabetic drugs were given as needed.

After 5 days of anticoagulant therapy with heparin patients were started on oral anticoagulants. All the patients were followed every week for 6 weeks.

\section{Relief of chest pain (Fig-1)}

\section{Results}

Chest pain subsided on the second day in 26 patients $(52 \%)$ on standard heparin and in 33 patients $(66 \%)$ treated with LMWHs. It subsided on the $3^{\text {rd }}$ day in 19 patients $(38 \%)$ treated with SH and $16(32 \%)$ treated with LMWHs. 2 patients $(4 \%)$ treated with standard heparin and $1(2 \%)$ with LMWHs showed relief of chest pain only on the fourth day. Recurrence of angina was seen in $3(6 \%)$ male patients on the fourth day of heparin therapy. No such recurrence was seen in patients treated with LMWHs

Bleeding tendencies (Fig-2)

Among 50 patients treated with conventional heparin $2(4 \%)$ patients developed haematemesis on the second day of treatment and haemoptysis occurred in $1(2 \%)$ patient on the third day. The remaining $47(94 \%)$ patients improved and were subsequently discharged. One patient who developed haematemesis with standard heparin was started on low molecular weight heparin on the third day and continued for 5 days. There were no adverse effects with low molecular weight heparin.

In patients treated with LMWHs subcutaneous ecchymosis was seen in $2(4 \%)$ cases. No major bleeding complications like haematemesis and haemoptysis occurred. There were no history acid peptic diseases or breathlessness during therapy with standard heparin and low molecular weight heparin.

ECG changes were studied serially and reduction in the ST elevation was seen on the second day in 30 patients $(60 \%)$ on standard heparin and $32(64 \%)$ patients on low molecular weight heparin. Changes occurred on the third in the remaining patients [Fig-3]

Improvement in the left ventricular function.

Improvement in the ejection fraction of left ventricle was also noted in the echocardiogram

The blood counts, showed no significant change after therapy with both heparin and low molecular heparins.

\section{Readmission}

4 patients $(8 \%)$ who were treated with standard heparin got readmitted after a period of two weeks and were treated with LMWHs for five days and showed improvement in their condition.

\section{Summary And Conclusion}

A Comparative study of conventional heparin and subcutaneous low molecular weight heparin was carried out in 100 patients admitted in the department of Cardiology, Government Hospital, Guntur with symptoms of acute myocardial infarction and unstable angina.

Group I: Consisted of 50 patients who received conventional heparin in conventional doses of 10000 units stratum intravenously followed by 5000 units intravenously $6^{\text {th }}$ hourly for five days.

Group II: Consisted of patients treated with low molecular weight heparin (enoxaparin) subcutaneously twice daily in a dose of 3500 units.

Both groups were eventually matched with regard to age, sex and risk factors and adjunctive drug therapy.

The study period consisted of four weeks follow up from the time of admission. The clinical end points considered at the end of five days were relief of chest pain recurrence of angina and occurrence of complications. The study also included changes in the ECG pattern improvement left ventricular function as evidenced by echocardiograms. Both the group patients were observed for bleeding complications both clinically and by lab methods

Recurrence of angina occurred in $6 \%$ of patients on standard heparin while no such recurrence occurred in patients on low molecular heparin. Bleeding complications (haematemesis in $4 \%$ and haemoptysis $2 \%$ ) occurred in patients on standard heparin while no such effects were seen in patients on low molecular heparin suggesting that low molecular heparin had lesser bleeding complications.

It is concluded from this study that low molecular weight heparin is equally effective in the treatment of acute myocardial infarction and unstable angina as that of conventional heparin with the added advantage of 
low complication rate and ease of administration and no necessity of maintaining the aPTT levels during therapy. The only disadvantage being the cost factor for low molecular heparin.

\section{References}

[1]. Gurfinkel et al, Low molecular heparin versus regular heparin or aspirin in the treatment of unstable angina and silent ischemia J.AM.Coll. Cardiol. 1995, 26:313-318.

[2]. Theroux, P. Ouimet, H Mccans J.et al., Aspirin, Heparin or Both to treat unstable angina. N.E.J.M. 1988. $319:$ 1105

[3]. Basu et al.,A prospective study of the value of monitoring heparin treatment with the activated partial thromboplastin time. N.E.J.M., 1972, 287: 324.

[4]. Cohen et al., A comparison of low molecular weight heparin with unfraction heparin for unstable CAD.N.E.J.M. 1997, 337: 447-52

[5]. FRISC Study group, Low molecular weight heparin during in stability in coronary artery disease Lancet: 1996: 347, 61-568.

[6]. Suvarna, T.T. Parikh, J.A. et al, Comparison of clinical outcome of fixed dose subcutaneous low molecular weight heparin (tinzaparin) with conventional heparin in unstable angina: a pilot study Indian Heart Journal 1997-49:159-162.

\section{Figure-1}

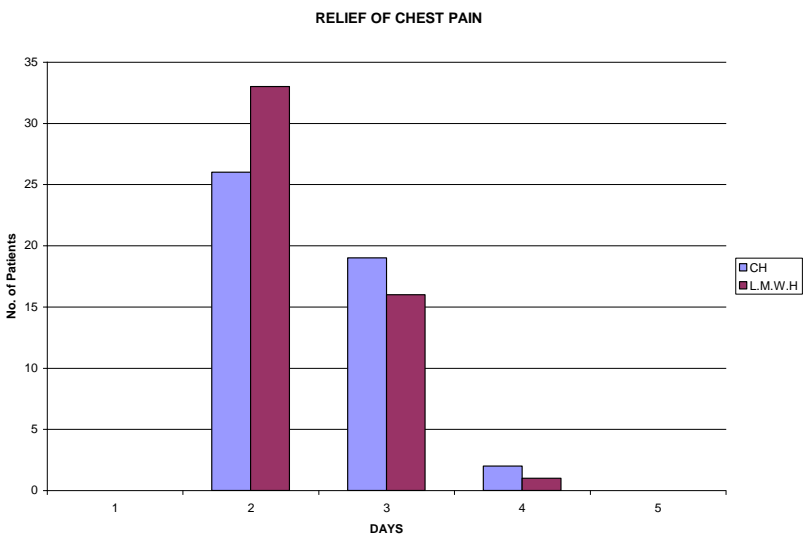

Figure-2 Bleeding Tendencies

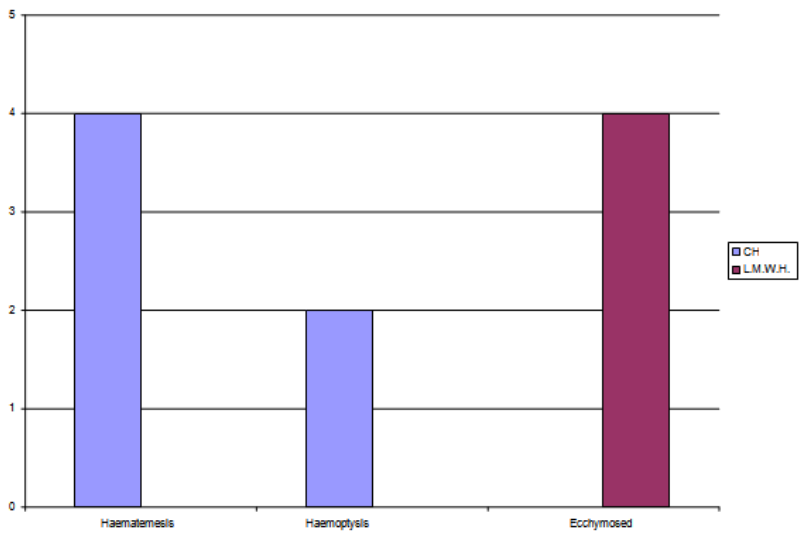

Figure-3

E.c.G. CHANGES

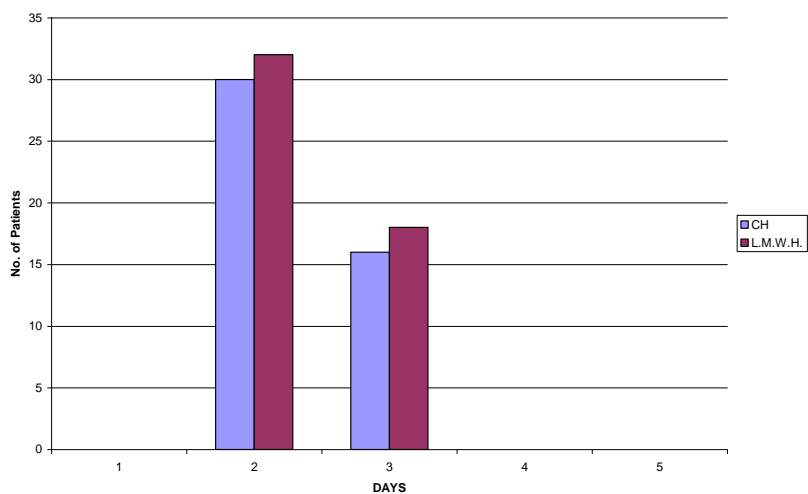

